\title{
L'adéquation et la transparence doivent primer sur la simplification
}

\section{Urs Stoffel}

Dr méd., membre du Comité central de la FMH, responsable du département Médecine et tarifs ambulatoires

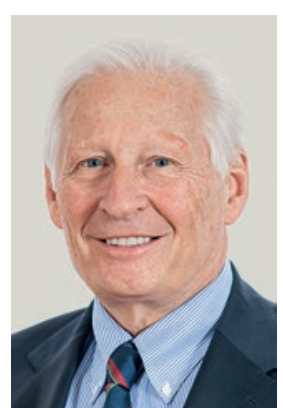

Régulièrement, différentes voix se font entendre pour demander la simplification du tarif à la prestation TARDOC et souligner, dans la foulée, les avantages des forfaits pour le secteur ambulatoire. Entretemps, plus personne ne conteste cependant la nécessité d'un tarif à la prestation comme fondement pour toutes les prestations médicales ambulatoires. L'art. 43, al. 4, LAMal, exige à cet égard que le tarif soit "[fixé] d'après les règles applicables en économie d'entreprise et [structuré] de manière appropriée». Un tarif exclusivement fondé sur le temps consacré à la prestation ne permet pas non plus de garantir le caractère approprié exigé par la loi.

Ce sont précisément ces critères qui ont guidé les partenaires tarifaires FMH, curafutura et CTM au cours de ces dernières années pour développer et négocier le TARDOC. Ce dernier étant entièrement nouveau, il n'est plus possible de comparer directement les différentes positions du TARMED avec les nouvelles positions du TARDOC.

Les partenaires tarifaires sont en ce moment en train de revoir la structure du TARDOC sur la base du rapport d'évaluation de l'OFSP. La structure tarifaire remaniée devra être déposée par l'ensemble des partenaires tarifaires d'ici la fin de l'année. La FMH, curafutura et la CTM ont profité de l'occasion pour analyser le potentiel de simplification du TARDOC. Or, il s'avère que dans de

\section{Le degré de détail de la structure tarifaire TARDOC permet un décompte approprié et transparent.}

nombreux cas, la simplification aurait lieu au détriment du caractère approprié et de la transparence, deux points qui revêtent tout autant d'importance que la simplification aux yeux des partenaires tarifaires. Le degré de détail de la structure tarifaire présente l'avantage de pouvoir clairement définir les règles d'application et de facturation et les valeurs intrinsèques qualitatives. Il en va de même des minutages des prestations à l'acte, qui peuvent être établis de manière plus précise. Ces avantages ne doivent pas être abandonnés au profit d'une simplification inadéquate. En revanche, un certain degré de simplification devrait être atteint dans le domaine des prestations chirurgicales.

Pour renforcer les soins médicaux de base, un chapitre entièrement consacré à la médecine de famille, respectant les exigences de l'art. 117a Cst., a été créé dans le TARDOC. A titre d'exemple, les travaux connexes sont beaucoup plus importants pour les médecins de famille que pour d'autres spécialistes, notamment en ce

«Aussi simple que possible, mais aussi différencié que nécessaire»: voilà le principe qui doit primer.

qui concerne la prise en charge de personnes âgées impliquant les proches et les soins à domicile. Un chapitre dédié permet ainsi de tenir compte de cette différence en toute transparence. Idem pour les soins palliatifs. Le cumul de prestations à l'acte et au temps est un instrument de tarification central dans le TARDOC. Pour cela, on a veillé à définir clairement les contenus de la prestation de base et de la prestation additionnelle. Si des situations critiques en matière de facturation devaient apparaître lors de l'application, elles seront identifiées et corrigées de manière ciblée grâce au monitorage.

A l'ère du numérique, avec une facturation qui se fait toujours plus par voie électronique dans les cabinets et avec le contrôle électronique des factures par les assureurs, il ne serait pas opportun de faire primer la simplification sur la transparence et l'adéquation. La facture moyenne d'un cabinet est inférieure à CHF 300, comprend un peu plus qu'une séance et est donc facile à comprendre pour la plupart des patients.

"Aussi simple que possible, mais aussi différencié que nécessaire»: voilà le principe qui doit primer aujourd'hui. Et c'est exactement ce que le TARDOC met en œuvre de façon cohérente. 